\title{
Factores perinatales asociados a la mortalidad neonatal en recién nacidos de muy bajo peso: estudio multicéntrico
}

\author{
Perinatal factors associated with neonatal mortality in \\ very low birth weight infants: a multicenter study
}

\author{
Dr. Carlos Grandi ${ }^{a}$, Dra. Agustina González ${ }^{b}$, Dr. José Zubizarretac $y$ \\ Red Neonatal NEOCOSUR
}

\section{RESUMEN}

Introducción. Objetivos. Evaluar los factores de riesgo asociados a la mortalidad neonatal en recién nacidos de muy bajo peso (RNMBP, $\leq$ $1500 \mathrm{~g}$ ) y el impacto de la administración antenatal de corticoides.

a. Investigador independiente, Consejo de Investigación en Salud, Maternidad Sardá, Buenos Aires, Argentina.

b. Hospital San José, Santiago, Chile.

c. Division of Decision, Risk and Operations, Affiliated Faculty, Statistics Department. Graduate School of Business, Columbia University, United States.

d.Miembros de la Red Neonatal NEOCOSUR (véase en Anexo en formato electrónico).

Correspondencia: Dr. Carlos Grandi, cgrandi@intramed.net

Financiamiento: El presente trabajo de investigación fue realizado con el apoyo del programa de becas "Ramón CarrilloArturo Oñativia", otorgadas por el Ministerio de Salud de la Nación, a través de la Comisión Nacional Salud Investiga.

Conflicto de intereses: Ninguno que declarar.

Recibido: 29-2-2016 Aceptado: 9-5-2016 Publicado Primero en Internet: 25-8-2016
Población y métodos. Análisis retrospectivo de una cohorte de RNMBP de 26 centros perinatales terciarios y universitarios de la Red Neonatal Sudamericana (NEOCOSUR), que incluye Argentina, Brasil, Chile, Paraguay, Perú y Uruguay, entre 2000 y 2011, y que cuenta con 11455 registros. Las características maternas, neonatales y la morbilidad se compararon entre los RNMBP que murieron y los sobrevivientes hasta el alta. Las variables asociadas con la muerte neonatal se determinaron mediante regresión logística.Se estimó el efecto del corticoide prenatal sobre la morbimortalidad neonatal utilizando el método de pareamiento.

Resultados. La tasa de mortalidad neonatal fue de $22,3 \%$ con una elevada variabilidad entre los centros. Los factores independientemente asociados a menor mortalidad de los RNMBP fueron la administración de corticoides prenatal (OR 0,49; IC 95\%: 0,43-0,54), mejor puntaje Z del peso de nacimiento (OR 0,63; 0,61-0,65), hipertensión arterial (OR 0,67;0,58-0,77) y cesárea (OR 0,75; 0,65-0,85). Mediante pareamiento, el riesgo de muerte se redujo en $38 \%$ asociado a los corticoides prenatales.

Conclusiones. Se identificaron importantes factores perinatales asociados con la mortalidad neonatal en RNMBP y se demostró el impacto de la administración de corticoides prenatales en la Red NEOCOSUR.

Palabrasclave: mortalidad neonatal, recién nacido de muy bajo peso, prematuro, factores de riesgo, estudios multicéntricos.

http:/ /dx.doi.org/10.5546/aap.2016.426

\section{INTRODUCCIÓN}

La prematuridad (edad gestacional $[E G]<37^{+0}$ semanas) es la principal causa directa de muerte de recién nacidos ( $R N)$, sobre todo en los de muy bajo peso de nacimiento
$(\mathrm{RNMBP}, \leq 1500 \mathrm{~g}) \cdot{ }^{1}$ La prematurez es también una importante causa indirecta de muerte, y la pobreza está fuertemente asociada con un mayor riesgo, lo que lleva a que cerca de $99 \%$ de las muertes neonatales mundiales se originen en países de bajos y medianos ingresos. ${ }^{2}$

Además, más de la mitad de aquellos que sobreviven al alta hospitalaria lo hacen con complicaciones graves, muchas de ellas crónicas y que afectan la calidad de vida del individuo y su familia, y demandan una cantidad importante de recursos médicos.

Es necesaria información sobre los factores de riesgo (FR) que llevan a estos desenlaces para priorizar intervenciones y planificar su desarrollo, evaluar la efectividad de intervenciones dirigidas específicamente hacia los FR y apreciar las tendencias a través del tiempo a nivel nacional e internacional., ${ }^{3,4}$

Aunque se dispone de resultados de RNMBP de países desarrollados, pocos estudios han examinado la morbimortalidad de RNMBP en Latinoamérica. ${ }^{5,6}$ Una de las estrategias para enfrentar este problema es que los centros de atención neonatal se incorporen a redes temáticas colaborativas especializadas, que, entre otros beneficios, permiten unificar criterios, optimizar recursos, comparar centros e identificar las prácticas médicas más beneficiosas (benchmarking). ${ }^{7}$

El presente estudio muestra la consolidación de la Red Neonatal Sudamericana (NEOCOSUR), que, 
desde hace 15 años, mantiene una monitorización continua de unidades de cuidados intensivos neonatales de 6 países de América del Sur (Argentina, Brasil, Chile, Paraguay, Perú y Uruguay).

Por tal razón, los objetivos del presente estudio fueron estudiar los FR asociados independientemente a la mortalidad de RNMBP nacidos en las unidades de Neonatología de la Red NEOCOSUR y evaluar el impacto de la administración antenatal de corticoides.

La hipótesis fue que la administración prenatal de corticoides disminuía la mortalidad neonatal y seleccionadas morbilidades de $\mathrm{RN} \leq 1500$ gramos.

\section{POBLACION Y MÉTODOS}

Se realizó un análisis retrospectivo de una cohorte multicéntrica de $\mathrm{RN}$ vivos con peso de nacimiento (PN) entre 500 y $1500 \mathrm{~g}$ de la Red NEOCOSUR. Los criterios de inclusión fueron EG igual o mayor de $24^{+0}$ semanas de gestación y PN entre 500 y $1500 \mathrm{~g}$. Los criterios de exclusión fueron muerte en sala de partos, $\mathrm{RN}$ derivado de otra institución y falta del registro de la mortalidad hasta el alta.

Los datos se recogieron de forma prospectiva y sistemática en 26 unidades entre el 1 de enero de 2000 y el 31 de diciembre de 2011, con definiciones rigurosas de cada evento. La información materna y neonatal de los RNMBP de cada centro fue ingresada en línea a la base de datos electrónica de la Unidad de Base de Datos. ${ }^{8}$

Las variables de resultado fueron la mortalidad y morbilidades neonatales al momento del egreso hospitalario. Las variables independientes fueron edad materna al momento del parto, nivel de educación, control prenatal, embarazo gemelar, diabetes, hipertensión arterial, corticoides prenatales (tratamiento completo, incompleto o ausente), terminación del embarazo (vaginal, cesárea con o sin trabajo de parto), sexo (masculino/femenino), EG (semanas completas al momento del parto) por fecha de la última menstruación (FUM) o ecografía precoz ( $1^{\text {er }}$ trimestre), PN (gramos), puntaje $\mathrm{Z}$ del PN, ${ }^{9}$ puntaje de Apgar 0-3 a los 5 minutos de vida, reanimación en sala de partos y tipo de centro (público/privado). Las morbilidades fueron síndrome de dificultad respiratoria (SDR: enfermedad de membrana hialina), asistencia respiratoria mecánica y número de días, presión positiva continua en la vía aérea (continuous positive airway pressure; CPAP, por sus siglas en inglés) en días, surfactante (y número de dosis), displasia broncopulmonar (DBP: oxígeno más de 28 días), ductus arterioso permeable hemodinámicamente significativo (DAP), sepsis precoz $(<72 \mathrm{~h})$ con hemocultivos positivos, enterocolitis necrotizante (ECN: grado II o mayor según Bell), hemorragia intracraneana (HIC: grado III o IV según Papile), leucomalacia periventricular (LPV), combinación de complicaciones mayores (CCM, dos o más de las siguientes entidades: DBP-HIC grados 3-4, ECN grados 2-3) y estadía (días).

La prematurez se definió según la Organización Mundial de la Salud (OMS) y la International Statistical Classification of Diseases and Related Health Problems $10^{\text {th }}$ Revision (ICD 10) y se clasificó en prematurez extrema $\left(<28^{+0}\right.$ semanas), muy prematuro $\left(28^{+0}-31^{+6}\right.$ semanas $)$ y prematurez moderada $\left(32^{+0}-36^{+6}\right.$ semanas de gestación). ${ }^{10}$

Para el cálculo del puntaje $Z$ del PN, se empleó una referencia poblacional argentina. ${ }^{9}$ Pequeño para la EG (PEG) y grande para la EG (GEG) se definieron siguiendo a Gruenwald ${ }^{11}$ según que el peso para la EG y el sexo estuvieran menos o más dos puntajes $Z$ de la mediana, respectivamente. Un puntaje $Z$ de +2 corresponde al percentil 97 y un puntaje $Z$ de -2 , al percentil 3 .

Los puntajes $Z$ se presentan para la población total y para los RN entre $24^{+0}$ y $32^{+6}$ semanas. PEG y GEG solo se presentan de $24^{+0}$ a $32^{+6}$ semanas con el fin de evitar la sobrerrepresentación de las dos condiciones, debido a que el límite de entrada en la base de datos está determinado por un peso al nacer hasta $1500 \mathrm{~g}$.

En las comparaciones, se incluyó el puntaje predictor de mortalidad para el RNMBP desarrollado por NEOCOSUR. ${ }^{12}$ Sucintamente, el puntaje NEOCOSUR estima la probabilidad de muerte de un RNMBP considerando peso al nacer, EG, puntaje de Apgar al quinto minuto, malformaciones congénitas con riesgo vital, uso prenatal de corticoides y sexo. Para el análisis de tendencia de la mortalidad, se empleó un modelo de regresión lineal.

La estadía se definió como los días desde el nacimiento hasta el alta. A efectos del análisis, las muertes fueron censuradas, de manera similar a los estudios de supervivencia.

Se calcularon medidas de tendencia central (media, mediana y proporciones) y medidas de dispersión (desvío estándar, intervalo de confianza [IC] al 95\% de la proporción e intervalo intercuartil [IIC]). El test U de Mann-Whitney y el test $t$ de Student se utilizaron para la comparación de variables continuas. El test de chi ${ }^{2}$ con corrección 
de Yates se empleó para la comparación de variables categóricas.

El análisis de regresión logística múltiple se empleó para explorar el efecto independiente de diversas condiciones sobre la mortalidad neonatal. Los modelos se ajustaron para centros públicos o privados, edad materna, embarazo múltiple, trastornos hipertensivos maternos, tratamiento prenatal con esteroides, tipo de parto, puntuación de Apgar a los 5 minutos $<3$, sexo, EG, puntaje $Z$ de PN y sepsis precoz probada. El grupo sobreviviente hasta el alta se estableció como grupo de referencia. Los resultados se presentan como odds ratio ajustados (ORa) con su IC al 95\% y se estimó la calibración de los modelos mediante el test de HosmerLemeshow y su capacidad de discriminación mediante curvas ROC.

\section{Pareamiento (matching) según uso prenatal de corticoides}

Se estimó el efecto de la administración prenatal de corticoides sobre la mortalidad neonatal y morbilidades en RNMBP empleando el método de pareamiento, que consiste en grupos idénticos en todo sentido salvo en el tratamiento. Los métodos de matching o pareamiento permiten construir dos muestras lo más parecidas posible en las covariables observadas, a semejanza de la estructura de un experimento aleatorizado. ${ }^{13}$
Un valor $P$ menor de 0,05 fue considerado como indicador de significación estadística. Se utilizaron los programas Statistica 9,0 (Statsoft, Tulsa, OK, USA), Stata 12 (Collage Station, Texas, USA) y EPIDAT 2,0 (OPS/OMS y Xunta de Galicia).

El estudio fue autorizado por el Comité de Ética del Hospital Materno Infantil Ramón Sardá, y se mantuvo el anonimato tanto de los centros como de los pacientes, atento a que solamente se incorporó el código de cada uno.

\section{RESULTADOS}

De 11991 RNMBP ingresados a la base de datos entre 2000 y 2011 (12 años), 11455 reunieron los criterios de selección (Figura 1).

La tasa de mortalidad neonatal (TMN) global del período fue de 22,3\% (IC 95\%: 21,6-23,1), y se observó una tendencia creciente $(\mathrm{p}=0,441$, Figura 2). Sin embargo, la mortalidad ajustada por riesgo (puntaje NEOCOSUR) disminuyó 60\% durante el período de estudio, aunque con una elevada variabilidad entre los diferentes centros.

La supervivencia global hasta el alta hospitalaria fue del $77,7 \%$, mientras que la supervivencia específica estuvo directamente relacionada con la EG, desde $54 \%$ para los prematuros extremos, $85,8 \%$ para los muy prematuros y $90,6 \%$ para los prematuros moderados ( $p<0,001)$.

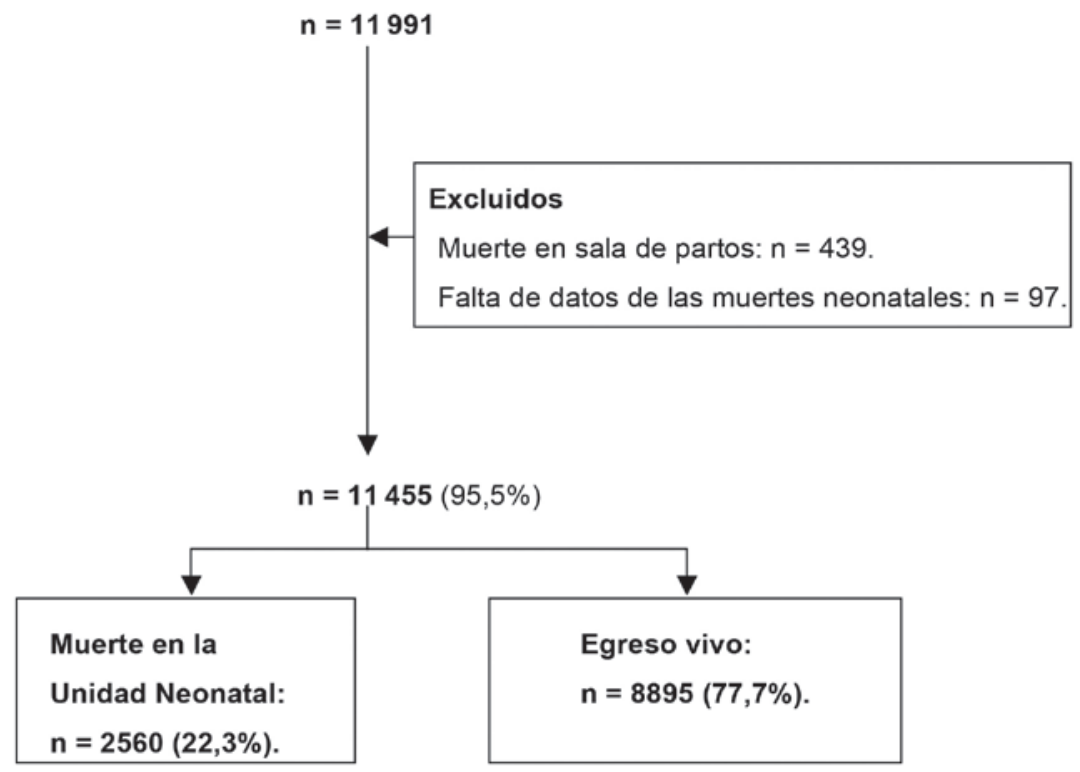


Los resultados de la morbilidad muestran los mismos grados de variabilidad que la mortalidad según los centros (datos no presentados).

Las madres de los niños fallecidos presentaron mayor frecuencia de adolescencia y cesárea con trabajo de parto, en comparación con los sobrevivientes $(\mathrm{p}<0,001)$ (Tabla 1$)$.

En los RNMBP fallecidos, predominó el sexo masculino y presentaron menor EG, PN, puntaje $Z$ del PN y PEG en comparación con los niños sobrevivientes. En contraste, presentaron casi el triple de frecuencia de prematuros extremos, mayor depresión al nacer y, consecuentemente, el doble de necesidad de reanimación en sala de partos ( $\mathrm{p}<0,001)$ (Tabla 2).

En la Tabla 3, se puede apreciar que, en comparación con los niños sobrevivientes, los RNMBP fallecidos presentaron un puntaje predictor de mortalidad al nacer 3 veces superior, mayor frecuencia de SDR, ventilación mecánica,

FigURA 2. Evolución de la tasa de mortalidad neonatal (NECOSUR, 2000-2011)

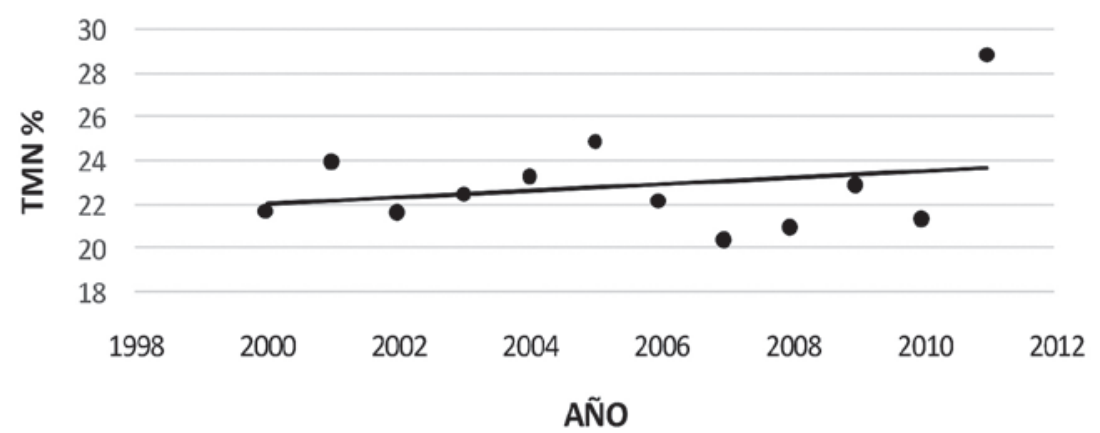

TMN: tasa de mortalidad neonatal.

TABLA 1. Comparación de las características demográficas, del embarazo y del parto entre fallecidos y sobrevivientes (NEOCOSUR, 2000-2011; $n=11455$ )

\begin{tabular}{|c|c|c|c|}
\hline Característica & $\begin{array}{c}\text { Fallecidos }(\mathrm{N}=2560) \\
n / N^{\mathrm{b}}\end{array}$ & $\begin{array}{c}\text { Sobrevivientes }(\mathrm{N}=8895) \\
n / N^{\mathrm{b}}\end{array}$ & $P^{a}$ \\
\hline Edad materna (años) ${ }^{c}$ & $27,0(7,4)$ & $27,8(7,3)$ & $<0,001$ \\
\hline$<16^{\mathrm{d}}$ & $54(2,1)$ & $198(2,2)$ & $<0,001$ \\
\hline $16-19$ & $431(16,9)$ & $1135(12,8)$ & \\
\hline $20-35$ & $1663(65,2)$ & $6025(67,8)$ & \\
\hline$>35$ & $400(15,7)$ & $1519(17,1)$ & \\
\hline Educación secundaria $^{\mathrm{d}}$ & $775 / 1733(44,7)$ & $2930 / 6031(48,6)$ & $<0,001$ \\
\hline Control prenatal ${ }^{\mathrm{d}}$ & $2013 / 2512(80,1)$ & $7856 / 8796(89,3)$ & $<0,001$ \\
\hline Embarazo múltiple $^{\mathrm{d}}$ & $414 / 2532(16,3)$ & $1760 / 8882(19,9)$ & $<0,001$ \\
\hline Diabetes $^{\mathrm{d}}$ & $59 / 2560(2,3)$ & $279 / 8895(3,1)$ & 0,028 \\
\hline Hipertensión ${ }^{\mathrm{d}, \mathrm{e}}$ & $451 / 2548(17,7)$ & $2778 / 11644(31,3)$ & $<0,001$ \\
\hline Parto vaginal $^{\mathrm{d}}$ & $1040 / 2556(40,6)$ & $2287 / 8889(25,7)$ & $<0,001$ \\
\hline Cesárea sin $\mathrm{TP}^{\mathrm{d}}$ & $883 / 2557(34,5)$ & $4574 / 8889(51,4)$ & $<0,001$ \\
\hline Cesárea con TPd & $633 / 2557(24,7)$ & $2028 / 8889(22,8)$ & 0,040 \\
\hline Corticoides prenatales $^{\mathrm{d}}$ & $1596 / 2523(63,2)$ & $7052 / 8773(80,3)$ & $<0,001$ \\
\hline Ninguno & $927(36,7)$ & $1721(19,6)$ & $<0,001$ \\
\hline Incompleto & $509(20,1)$ & $1683(19,1)$ & \\
\hline Completo & $1049(41,5)$ & $5119(58,3)$ & \\
\hline$>1$ serie & $38(1,5)$ & $250(2,8)$ & \\
\hline
\end{tabular}

TP: trabajo de parto.

a Test de Student o chi ${ }^{2}$ entre fallecidos y sobrevivientes.

${ }^{\mathrm{b}}$ Cuando se especifica el denominador, indica que pueden faltar datos.

${ }^{\mathrm{c}}$ Media (DE).

${ }^{\mathrm{d}} \mathrm{n}(\%)$.

e Hipertensión crónica más inducida por el embarazo. 
necesidad de surfactante, DBP, hemorragia intraventricular (HIV), ECN, DAP, sepsis precoz $y$ el doble de puntaje combinado $(p<0,001)$.

Los FR independientemente asociados a mayor mortalidad fueron EG menor de 32 semanas, puntaje de Apgar $<3$ al $5^{\text {to }}$ minuto y sepsis precoz. Los factores independientemente asociados a menor mortalidad fueron la administración de corticoides prenatal, mejor puntaje Z del PN, hipertensión en el embarazo y cesárea (Tabla 4).

TABLA 2. Comparación de las características neonatales entre fallecidos y sobrevivientes (NEOCOSUR, 2000-2011; $n=11455$ )

\begin{tabular}{|c|c|c|c|}
\hline Característica & $\begin{array}{c}\text { Fallecidos }(\mathrm{N}=2560) \\
n / N^{\mathrm{b}}\end{array}$ & $\begin{array}{c}\text { Sobrevivientes }(\mathrm{N}=8895) \\
n / N^{\mathrm{b}}\end{array}$ & $P^{a}$ \\
\hline Sexo masculinoc & $1408 / 2552(55,1)$ & $4399 / 8889(49,0)$ & $<0,001$ \\
\hline Edad gestacional (s. $)^{\mathrm{d}}$ & $27,1(2,7)$ & $29,6(2,5)$ & $<0,001$ \\
\hline Prematurez extrema ${ }^{c}$ & $1528(59,6)$ & $1796(20,2)$ & $<0,001$ \\
\hline Muy prematuro ${ }^{c}$ & $803(31,3)$ & $4889(54,9)$ & \\
\hline Prematurez moderadac ${ }^{c}$ & $229(8,9)$ & $2210(24,8)$ & \\
\hline Peso de nacimiento $(\mathrm{g})^{\mathrm{d}}$ & $884(256)$ & $1163(240)$ & $<0,001$ \\
\hline Puntaje $\mathrm{Z}$ del peso de nacimiento ${ }^{\mathrm{d}}$ & $-0,88(1,8)$ & $-1,23(2,2)$ & $<0,001$ \\
\hline Puntaje $\mathrm{Z}$ del peso de nacimiento ${ }^{\mathrm{d}, \mathrm{e}}$ & $-0,59(1,2)$ & $-0,54(1,3)$ & 0,066 \\
\hline$P_{E G}{ }^{c, e}$ & $266 / 2331(11,4)$ & $1005 / 6685(15,0)$ & $<0,001$ \\
\hline $\mathrm{GEG}^{\mathrm{c}, \mathrm{e}}$ & $26 / 2331(1,11)$ & $70 / 6685(1,04)$ & 0,782 \\
\hline Apgar al $5^{\text {to }} \min <3^{c}$ & $268(10,4)$ & $114(1,28)$ & $<0,001$ \\
\hline Reanimación en sala partos ${ }^{c}$ & $2084 / 2543(81,9)$ & $4282 / 8827(48,5)$ & $<0,001$ \\
\hline
\end{tabular}

PEG: pequeño para la edad gestacional. GEG: grande para la edad gestacional.

a Test de Student o chi ${ }^{2}$ entre fallecidos y sobrevivientes.

${ }^{\mathrm{b}}$ Cuando se especifica el denominador, indica que pueden faltar datos.

${ }^{\mathrm{c}} \mathrm{n}(\%)$.

d Media (DE).

${ }^{\text {e }}$ Edad gestacional de 24-32 semanas.

TABLA 3. Morbilidades neonatales: fallecidos versus sobrevivientes (NEOCOSUR, 2000-2011; n= 11455)

\begin{tabular}{|c|c|c|c|}
\hline Característica & $\begin{array}{c}\text { Fallecidos }(\mathrm{N}=2560) \\
n / N^{\mathrm{b}}\end{array}$ & $\begin{array}{c}\text { Sobrevivientes }(\mathrm{N}=8895) \\
n / N^{\mathrm{b}}\end{array}$ & $P^{a}$ \\
\hline Puntaje NEOCOSUR ${ }^{c}$ & $0,459(0,27)$ & $0,149(0,17)$ & $<0,001$ \\
\hline $\mathrm{SDR}^{\mathrm{d}}$ & $2283 / 2532(90,1)$ & $6184 / 8876(69,6)$ & $<0,001$ \\
\hline Ventilación mecánica $^{\mathrm{d}}$ & $2361 / 2535(93,1)$ & $5027 / 8846(56,8)$ & $<0,001$ \\
\hline Ventilación mecánica (días)e & $3(9)$ & $1(6)$ & $<0,001$ \\
\hline $\mathrm{CPAP}$ (días) $^{\mathrm{e}}$ & 0 & $1(5)$ & $<0,001$ \\
\hline Surfactante & $1985 / 2512(79)$ & $4263 / 8580(49,6)$ & $<0,001$ \\
\hline Dosis de surfactante ${ }^{\mathrm{e}}$ & $1(1)$ & $0(1)$ & $<0,001$ \\
\hline $\mathrm{DBP}^{\mathrm{d}, \mathrm{f}}$ & $213 / 366(58,2)$ & $2461 / 7837(31,4)$ & $<0,001$ \\
\hline ECN grados $2-3^{\mathrm{d}}$ & $435 / 2527(17,1)$ & $853 / 8858(9,6)$ & $<0,001$ \\
\hline HIV grados $3-4^{\mathrm{d}}$ & $582 / 1694(34,3)$ & $511 / 8595(5,9)$ & $<0,001$ \\
\hline LMPd $^{d}$ & $95 / 2245(4,2)$ & $470 / 8752(5,3)$ & 0,029 \\
\hline $\mathrm{DAP}^{\mathrm{d}}$ & $1060 / 2488(42,6)$ & $3022 / 8835(34,2)$ & $<0,001$ \\
\hline Sepsis precoz ${ }^{\mathrm{d}}$ & $180 / 2484(7,2)$ & $233 / 8818(2,6)$ & $<0,001$ \\
\hline $\mathrm{CCM}^{\mathrm{d}}$ & $2160 / 2560(84,3)$ & $4808 / 8895(54,0)$ & $<0,001$ \\
\hline Duración de la internación (días)e & $6(14)$ & $54(37)$ & $<0,001$ \\
\hline
\end{tabular}

SDR: síndrome de dificultad respiratoria; CPAP: presión continua de la vía aérea; DBP: displasia broncopulmonar;

ECN: enterocolitis necrotizante; HIV: hemorragia intraventricular; LMP: leucomalacia periventricular;

DAP: ductus arterioso persistente; CCM: combinación de complicaciones mayores.

a Test de Student, Mann-Whitney o chi ${ }^{2}$ entre fallecidos y sobrevivientes.

${ }^{\mathrm{b}}$ Cuando se especifica el denominador, indica que pueden faltar datos.

${ }^{\mathrm{c}}$ Media (DE).

$\mathrm{d} n(\%)$.

${ }^{\text {e }}$ Mediana (IIQ).

${ }^{\mathrm{f}}$ Internación $>28$ días. 
El ajuste del modelo fue satisfactorio (HosmerLemeshow, $\mathrm{p}=0,147$ ) y el área bajo la curva ROC fue de 0,825 .

No se observaron diferencias en la TMN según el volumen de RN internados. La TMN del sector público ascendió al 23,2\%, mientras que la del sector privado fue de 19,8 (OR 1,22; IC 95\%: 1,101,36; $\mathrm{p}<0,001)$.

La frecuencia de combinación de complicaciones mayores (CCM) al momento del alta hospitalaria varió de $42,8 \%$ a $74,3 \%$ entre los 26 centros, con una media de 60,8\% (IC 95\%: 59-61). De acuerdo con el origen del centro, la CCM fue 59,3\% (58-60) para los públicos y $57,7 \%$ (56-59 para los privados) $(\mathrm{p}=0,121)$.

La mediana de la estadía fue de 47 días (IIC: 41 días), y se observaron diferencias importantes entre los diferentes centros, similares a las observadas en mortalidad y morbilidad.

Pareamiento: La muestra final luego del pareamiento incluyó 4192 RN (2096 en cada rama). Aquellos RNMBP cuyas madres recibieron esteroides prenatales mostraron un $38 \%$ de reducción del riesgo de morir en comparación

TABLA 4. Factores asociados a la mortalidad en recién nacidos de muy bajo peso: análisis por regresión logística múltiple (NEOCOSUR, 2000-2011; $n=11455$ )

\begin{tabular}{|c|c|c|}
\hline $\begin{array}{l}\text { Variable } \\
\text { (IC 95\%) }\end{array}$ & OR ajustado & $P$ \\
\hline Centro público & $1,104(0,975-1,249)$ & 0,117 \\
\hline Edad materna & $1,000(0,993-1,007)$ & 0,918 \\
\hline Embarazo múltiple & $0,901(0,785-1,032)$ & 0,133 \\
\hline Hipertensión & $0,674(0,585-0,775)$ & $<0,001$ \\
\hline Corticoides prenatales & $0,492(0,439-0,540)$ & $<0,001$ \\
\hline Cesárea sin $\mathrm{TP}^{\mathrm{b}}$ & $0,751(0,658-0,858)$ & $<0,001$ \\
\hline Cesárea con $\mathrm{TP}^{\mathrm{b}}$ & $0,842(0,737-0,963)$ & 0,019 \\
\hline Apgar al $5^{\text {to }} \min <3$ & $5,496(4,258-7,093)$ & $<0,001$ \\
\hline Sexo masculino & $0,905(0,819-1,001)$ & 0,058 \\
\hline Edad gestacional & $0,571(0,559-0,590)$ & $<0,001$ \\
\hline Prematurez extrema ${ }^{c}$ & $38,71(29,53-50,74)$ & $<0,001$ \\
\hline Muy prematuro ${ }^{c}$ & $6,905(5,369-8,881)$ & $<0,001$ \\
\hline $\begin{array}{l}\text { Puntaje } \mathrm{Z} \text { del peso } \\
\text { de nacimiento }\end{array}$ & $0,633(0,612-0,656)$ & $<0,001$ \\
\hline Sepsis precoz & $2,474(1,961-3,121)$ & $<0,001$ \\
\hline
\end{tabular}

OR: odds ratio. IC: intervalo de confianza.

a Ajustado para centro público, edad materna, embarazo múltiple, hipertensión materna, corticoides prenatales, tipo de parto, puntaje de Apgar al $5^{\text {to }} \min <3$, sexo, edad gestacional, puntaje $\mathrm{Z}$ del peso de nacimiento y sepsis precoz.

${ }^{\mathrm{b}}$ Referencia: parto vaginal.

c Referencia: prematurez tardía. con aquellos cuyas madres no lo recibieron $(\mathrm{p}<0,001)$. Similares efectos se observaron para asfixia $($ OR 0,49$)$ y SDR $($ OR 0,62$)$. El resto de las morbilidades no mostró asociación con los corticoides prenatales (Tabla 5).

\section{DISCUSIÓN}

Este es el segundo estudio multicéntrico latinoamericano que explora los FR asociados a la mortalidad neonatal de RNMBP y el impacto de los corticoides prenatales en la prevención de resultados perinatales adversos. Es más actualizado y con mayor tamaño muestral. ${ }^{14}$

Se demostró que la administración de corticoides prenatales, mejor puntaje $\mathrm{Z}$ del PN, hipertensión arterial y cesárea eran factores independientemente asociados a menor mortalidad de los RNMBP y el impacto de los corticoides prenatales sobre la morbimortalidad neonatal.

La TMN global mostró una tendencia creciente en el período estudiado con una amplia variabilidad entre centros, de forma parecida a lo publicado. ${ }^{15}$ No obstante, la mortalidad ajustada por riesgo descendió notablemente, lo que remarcó la necesidad de ajustar los resultados al momento de analizar tendencias.

La EG ejerce la mayor influencia sobre el pronóstico de los RNMBP, y se observó un aumento progresivo de la supervivencia con el incremento de la EG.

Se identificaron cuatro grupos de factores asociados independientemente con un resultado desfavorable, a saber: aquellos asociados con la atención obstétrica (ausencia de exposición a corticoides prenatales, parto vaginal),

TABLA 5. Riesgos de diferentes resultados perinatales adversos asociados a la administración de corticoides prenatales (NEOCOSUR, 2000-2011; $n=4192$ recién nacidos)

\begin{tabular}{lccc} 
RESULTADO & ORa & IC 95\% & $\boldsymbol{P}$ \\
\hline Muerte & 0,62 & $0,52-0,74$ & $<0,001$ \\
Apgar al 5 to min $<3$ & 0,49 & $0,34-0,68$ & $<0,001$ \\
SDR & 0,62 & $0,53-0,74$ & $<0,001$ \\
Displasia broncopulmonar & 1,16 & $0,99-1,36$ & 0,063 \\
Hemorragia intraventricular & 1,07 & $0,88-1,30$ & 0,524 \\
Sepsis precoz & 0,90 & $0,66-1,21$ & 0,507 \\
CCM & 0,95 & $0,81-1,12$ & 0,544 \\
\hline
\end{tabular}

ORa: odds ratio ajustado. IC: intervalo de confianza. SDR: síndrome de dificultad respiratoria. CCM: combinación de complicaciones mayores. 
los relacionados con variables biológicas (sexo masculino, prematuridad de menos de 32 semanas, restricción del crecimiento intrauterino), los relacionados con la asistencia en el parto (puntuación de Apgar $<3$ al $5^{\text {to }}$ minuto) y los relacionadas con los cuidados intensivos (sepsis precoz).

Las características maternas de los RNMBP fallecidos ilustran la fuerte influencia del componente social y del acceso a la atención sobre los resultados perinatales, lo que concuerda con una reciente revisión sistemática. ${ }^{16}$

Es conocida la asociación entre hipertensión en el embarazo y reducción del tamaño al nacer, que puede acelerar la maduración pulmonar $\mathrm{y}$, consecuentemente, reducir la mortalidad neonatal $(\mathrm{MN}) \cdot{ }^{17}$ Aunque el tema es muy controvertido, algunos estudios retrospectivos indican que puede haber una reducción de la tasa de complicaciones relacionadas con la prematuridad cuando el niño nace por cesárea. ${ }^{18,19}$ Estos resultados resaltan lo imperativo de la pesquisa precoz de FR prenatales y una adecuada conducción del trabajo de parto y reanimación del RN en su relación entre mortalidad y EG, PN, restricción del crecimiento y asfixia al nacer.

La gran variabilidad intercentros se ha reportado en otros estudios ${ }^{20,21}$ y se puede explicar tanto por las características de la población de cada unidad y sus riesgos inherentes como por las intervenciones diagnósticas y terapéuticas realizadas en cada centro, en especial, la conducta de los profesionales frente a prematuros en el límite de la viabilidad. Otro aspecto fundamental para considerar es que América Latina tiene un grave déficit de enfermeras, que fue documentado en un estudio de NEOCOSUR. ${ }^{22}$

Los FR independientemente asociados con la mortalidad hallados coinciden con los descritos con anterioridad en varios modelos de predicción..$^{23,24}$

La información obtenida por las redes epidemiológicas permite estudiar las mejores estrategias para reducir la mortalidad y la morbilidad neonatal, basadas en procesos para mejorar la calidad de la atención perinatal. ${ }^{25}$ Así, la mortalidad reportada para los RNMBP en redes neonatales de países desarrollados (Euro Neo Net/Euro Peristat, ${ }^{26}$ Vermont-Oxford, ${ }^{27}$ Red Neonatal de Canadá, ${ }^{28}$ National Institute of Child Health and Human Development [NICHD], ${ }^{29}$ Sociedad Española de Neonatología) ${ }^{23}$ varía entre $12,4 \%$ y $28 \%$. En contraste, los resultados de la Red Brasilera de Investigación Neonatal, ${ }^{30}$ así como de la Red de Neonatología de los Hospitales Públicos de la Ciudad de Buenos Aires ${ }^{31}$ muestran resultados más desfavorables, asociados, especialmente, a la baja tasa de corticoides prenatales. Sin embargo, al momento de analizar estas diferencias, hay que tener presente el denominador que se utiliza para calcular la tasa de mortalidad y los criterios de inclusión que varían entre los estudios, ${ }^{32}$ además de diferencias poblacionales, prácticas perinatales y recursos.

El método de pareamiento mostró inequívocamente en esta amplia y variada población, con una cobertura de corticoides maternos del 76,5\% (IC 95\%: 75-77), su impacto sobre la MN ( $\mathrm{p}<0,001)$. Al restringir el análisis para los $\mathrm{RN}<35^{+0}$ semanas, que es la indicación actual de corticoides, el efecto protector aumentó levemente (ORa 0,47; IC 95\%: 0,42-0,53). Durante el período del estudio, el porcentaje de esteroides prenatales ha permanecido estable. Esto es similar a un estudio chileno, ${ }^{33}$ pero contrasta con un estudio brasilero, ${ }^{34}$ en el que apenas el 54\% recibió, por lo menos, una dosis, mientras que, en los EE. UU., hace más de 15 años que el 79\% de las gestantes reciben la medicación, ${ }^{35}$ cifra muy cercana al presente estudio.

Las diferencias en la estadía de los RNMBP no se deben solamente a complicaciones intrahospitalarias y se puede especular que pueden atribuirse a prácticas de egreso diferentes.

Entre las limitaciones del presente estudio, se destacan que se trata de un análisis retrospectivo, tiene sesgos y factores de confusión que no pudieron ser controlados (el modelo final explicó el $20 \%$ de la variabilidad de la MN), por lo que nuestros resultados pueden estar afectados, en cierta medida, por confusión residual. La población analizada representa entre el $8 \%$ y el $40 \%$ de los RNMBP a nivel nacional, por lo que los resultados no serían generalizables a toda la población.

Sin embargo, este estudio multicéntrico brinda datos sobre la muerte y la supervivencia con las principales morbilidades de los RNMBP prematuros de unidades hospitalarias públicas y privadas que son centros nacionales de referencia en los cuidados perinatales.

La principal fortaleza es el tamaño muestral, el carácter multicéntrico del estudio, que permitió una distribución geográfica, la recolección prospectiva y sistemática de los datos y la calibración y discriminación adecuadas del modelo final. 


\section{CONCLUSIONES}

Se estimó el riesgo independiente de diversas condiciones perinatales asociadas a la $\mathrm{MN}$ en RNMBP y se demostró la hipótesis del impacto de la administración de corticoides prenatales en la Red NEOCOSUR.

\section{Agradecimientos}

A todos los centros de la Red NEOCOSUR que participaron en este estudio (véase el Anexo) y a la Unidad de Base de Datos por la preparación de la base de datos (Dra. Ivonne D'Apremont).

\section{REFERENCIAS}

1. March of Dimes, PMNCH, Save the Children, WHO. Born too soon: The Global Action Report on Preterm Birth. Geneva: World Health Organization; 2012.

2. Lawn JE, Cousens S, Zupan J. 4 million neonatal deaths: When? Where? Why? Lancet 2005;365(9462):891-900.

3. Bryce J, Boschi-Pinto C, Shibuya K, Black R. WHO estimates of the causes of death in children. Lancet 2005;365(9465):1147-52.

4. Liu L, Johnson HL, Cousens S, Perin J, et al. Global, regional, and national causes of child mortality: an updated systematic analysis for 2010 with time trends since 2000. Lancet 2012;379(9832):2151-61.

5. Belizán JM, Cafferata ML, Belizán M, Tomasso G, et al. Goals in maternal and perinatal care in Latin America and the Caribbean. Birth 2005;32(3):210-8.

6. De Almeida MF, Guinsburg R, Martinez FE, Procianoy RS, et al. Perinatal factors associated with early deaths of preterm infants born in Brazilian Network on Neonatal Research centers. J Pediatr (Rio J) 2008;84(4):300-7.

7. Hummler H. Benchmarking in neonatal intensive care: obstetrical and neonatal practices and registration policies may influence outcome data. Arch Dis Child Fetal Neonatal Ed 2013;98(2):F96-7.

8. Fernández R, D’Apremont I, Domínguez A, Tapia JL, ET al. Supervivencia y morbilidad en recién nacidos de muy bajo peso al nacer en una Red Neonatal sudamericana. Arch Argent Pediatr 2014;112(5):405-12.

9. Urquia ML,Alazraqui M,SpinelliHG,FrankJW. Referencias poblacionales argentinas de pesoal nacer según multiplicad del parto, sexo y edad gestacional. Rev Panam Salud Publica 2011;29(2):108-19.

10. WHO: recommended definitions, terminology and format for statistical tables related to the perinatal period and use of a new certificate for cause of perinatal deaths. Modifications recommended by FIGO as amended October 14, 1976. Acta Obstet Gynecol Scand 1977;56(3):247-53.

11. Gruenwald P. Growth of the human fetus. I. Normal growth and its variation. Am J Obstet Gynecol 1966;94(8):1112-9.

12. Marshall G, Tapia JL, D'Apremont I, Grandi C, et al. A new score for predicting neonatal very low birth weight mortality risk in the NEOCOSURSouth American Network. J Perinatol 2005;25(9):577-82

13. Zubizarreta JR. Using mixed integer programming for matching in an observational study of acute kidney failure after surgery. J Am Stat Assoc 2012;107(500):1360-71.

14. Grupo Colaborativo Neocosur. Very-low-birth-weight infant outcomes in 11 South American NICUs. I Perinatol 2002;22(1):2-7.

15. Draper ES. Evaluating and comparing neonatal outcomes. Arch Dis Child Fetal Neonatal Ed 2010;95(3):F158-9.

16. Upadhyay RP, Krishnan A, RaiSK, Chinnakali P, et al. Need to focus beyond the medical causes: a systematic review of the social factors affecting neonatal deaths. Paediatr Perinat Epidemiol 2014;28(2):127-37.
17. Von Dadelszen P, Magee LA, Taylor EL, Muir JC, et al. Maternal hypertension and neonatal outcome among small for gestational age infants. Obstet Gynecol 2005;106(2):335-9.

18. Skupski DW, Greenough A, Donn SM, Arabin B, et al. Delivery mode for the extremely premature fetus: a statement of the prematurity working group of the World Association of Perinatal Medicine. J Perinat Med 2009;37(6):583-6.

19. Alfirevic Z, Milan SJ, Livio S. Caesarean section versus vaginal delivery for preterm birth in singletons. Cochrane Database Syst Rev 2012;6:CD000078.

20. Draper ES, Zeitlin J, Fenton AC, Weber T, et al. Investigating the variations in survival rates for very preterm infants in 10 European regions: the MOSAIC birth cohort. Arch Dis Child Fetal Neonatal Ed 2009;94(3):F158-63.

21. Field D, Smith L, Manktelow B, McParland P, et al. Interpretation of early life mortality rates. Arch Dis Child Fetal Neonatal Ed 2015;100(2):F99-100.

22. Grandi C, González A, Meritano I, Grupo Colaborativo Neocosur. Riesgo de morbimortalidad neonatal de recién nacidos menores de $1500 \mathrm{~g}$ asociado al volumen de pacientes, personal médico y de enfermería: una investigación multicéntrica latinoamericana. Arch Argent Pediatr 2010;108(6):499-510.

23. Moro M, Figueras-Aloy J, Fernández C, Doménech E, et al. Mortality for newborns of birthweight less than 1500 $\mathrm{g}$ in Spanish neonatal units (2002-2005). Am J Perinatol 2007;24(10):593-601.

24. Pollack MM, Koch MA, Bartel DA, Rapoport I, et al. A comparison of neonatal mortality risk prediction models in very low birth weight infants. Pediatrics 2000;105(5):1051-7.

25. Evans N, Hutchinson J, Simpson JM, Donoghue D, et al. Prenatal predictors of mortality in very preterm infants cared for in the Australian and New Zealand Neonatal Network. Arch Dis Child Fetal Neonatal Ed 2007;92(1):F34-40.

26. Euro Peristat. European Perinatal Health Report. Health and Care of Pregnant Women and Babies in Europe in 2010. [Acceso: 11 de mayo de 2016]. Disponible en: http:/ / www.europeristat.com/images/doc/Peristat $\% 202013 \% 20$ V2.pdf.

27. Horbar JD, Carpenter JH, Badger GJ, Kenny MJ, et al. Mortality and neonatal morbidity among infants 501 to 1500 grams from 2000 to 2009. Pediatrics 2012;129(6):1019-26.

28. Shah PS, Sankaran K, Aziz K, Allen AC, et al. Outcomes of preterm infants 29 weeks gestation over 10 -year period in Canada: a cause for concern? J Perinatol 2012;32(2):132-8.

29. Stoll BJ, Hansen NI, Bell EF, Shankaran S, et al. Neonatal outcomes of extremely preterm infants from the NICHD Neonatal Research Network. Pediatrics 2010;126(3):443-56.

30. Guinsburg R, de Almeida MF, de Castro JS, Silveira RC, et al. Death or survival with major morbidity in VLBW infants born at Brazilianneonatal research network centers.JMatern Fetal Neonatal Med 2016;29(6):1005-9.

31. Red deNeonatología delos Hospitales Públicos dela Ciudad Autónoma de Buenos Aires. Morbimortalidad de recién nacidos con menos de 1500 gramos asistidos en hospitales públicos de la ciudad de Buenos Aires. Arch Argent Pediatr 2012;110(5):394-403.

32. Guillen Ú, DeMauro S, Ma L, Zupancic J, et al. Survival rates in extremely low birthweight infants depend on the denominator: avoiding potential for bias by specifying denominators. Am J Obstet Gynecol 2011;205(4):329.e1-7.

33. Gonzalez R, Merialdi M, Lincetto O, Lauer J, etal. Reduction in neonatal mortality in Chile between 1990 and 2000. Pediatrics 2006;117(5):e949-54.

34. Azeredo Cardoso RC, Guimaraes Flores PV, Lima Vieira C, Vergetti Bloch $\mathrm{K}$, et al. Infant mortality in a very low birth weight cohort from a public hospital in Rio de Janeiro, RJ, Brazil. Rev Bras Saude Mater Infant 2013;13(3):237-46.

35. Fanaroff AA, Stoll BJ, Wright LL, Carlo WA, et al. Trends in neonatal morbidity and mortality for very low birthweight infants. Am J Obstet Gynecol 2007;196(2):147.e1-8. 


\begin{abstract}
ANEXO
El presente trabajo contó con la colaboración de los siguientes miembros de la Red NEOCOSUR:

\section{ARGENTINA}

Guillermo Colantonio, Jorge Zapata, Gastón Pérez, Liliana Rochinotti, Inés Galíndez, Luis Prudent (Clinica y Maternidad Suizo Argentina, Buenos Aires); Gonzalo Mariani, José María Ceriani, Silvia Fernández, Pablo Brener, Carlos Fustiñana † (Hospital Italiano, Buenos Aires); Liliana Roldán, Héctor Sexer, Gladys Sáa, Débora Sabatelli, Elizabeth Lombardo, María Laura Gendra, Paula Molina, Jorge Tavosnaska (Hospital Juan Fernández, Buenos Aires); Daniel Agost, Gabriela Torres, Jorge Ríos, Augusto Fischetti, Mónica Rinaldi (Hospital Lagomaggiore, Mendoza); Claudio Solana, Elio Rojas, Ricardo Nieto, Javier Meritano, Miguel Larguia, Carlos Grandi (Maternidad Sardá, Buenos Aires); Marcelo Decaro, Lionel Cracco, Gustavo Bassi, Noemí Jacobi, Edith Martínez, María San Miguel, Andrea Brum, Néstor Vain (Sanatorio de la Trinidad, Buenos Aires).
\end{abstract}

\title{
CHILE
}

Jorge Fabres, Alberto Estay, Álvaro González, Mariela Quezada, Soledad Urzúa, Javier Kattan (Hospital Clínico Pontificia Universidad Católica de Chile, Santiago); Solange Rojas, Sandra Vignes, Guillermo Marshall, Luis Villarroel (Unidad Base de Datos, Pontificia Universidad Católica, Santiago); Rodrigo Ramírez, María Eugenia Hübner, Jaime Burgos, Jorge Catalán (Hospital Clínico Universidad de Chile, Santiago); Lilia Campos, Roxana Aguilar, Sergio Treuer, Jimena Giaconi, Aldo Bancalari, Jorge León del Pedregal (Hospital Guillermo Grant, Concepción); Marisol Escobar, Viviana Veas, Daniela Sandino, Antonio Salvado, Alejandra Núñez, Jane Standen (Hospital Gustavo Fricke, Viña del Mar); Agustina González, Claudia Ávila, Ana Luisa Candia (Hospital San José, Santiago); Claudia Toro, Beatriz Milet, Angélica Alegría, Patricia Mena (Hospital Dr. Sótero del Río, Santiago).

\section{PARAGUAY}

Elizabeth Céspedes, Ramón Mir, Elvira Mendieta, Larissa Genes, José Lacarruba (Departamento de Hospital de Clínicas de Asunción).

\section{PERÚ}

Verónica Webb, Fabiola Rivera, Enrique Bambaren, Marilú Rospigliosi, Margarita Llontop, Jaime Zegarra (Hospital Cayetano Heredia, Lima).

\section{URUGUAY}

Sandra Gugliucci, Ana Lain, Alicia Prieto, Cristina Hernández, Mariza Martínez, Gabriela Bazán, Daniel Borbonet, Rubén Panizza + (Facultad de Medicina, Hospital Pereira Rossell, Servicio de Recién Nacidos, Montevideo). 